\title{
Hybrid repair for secondary lumbar hernias: Three cases with different etiologies
}

\author{
Dakan Kulaçoğlu, ${ }^{1}$ (D) Mehmet Özer ${ }^{2}$ \\ 'Department of General Surgery, Ankara Hernia Center, Ankara, Turkey \\ ${ }^{2}$ Department of General Surgery, Ankara Atatürk Training and Research Hospital, Ankara, Turkey
}

\begin{abstract}
Lumbar hernia is a rare type of abdominal wall hernias. Among acquired lumbar hernias, secondary cases are less common than spontaneous ones. Although infections can cause lumbar hernias, most of the secondary hernias develop following surgical procedures or different types of traumas. These types of hernia are treated in comply with the principles of incisional hernia treatment. Herein a case series of three consecutive patient with secondary lumbar hernias are presented. The etiology differs in each case. The hernias were repaired with hybrid technique (laparoscopic plus open) with no perioperative events.
\end{abstract}

Keywords: Hernia repair; hybrid repair; laparoscopy; lumbar hernia; mesh.

\section{Introduction}

Lumbar hernia is a rare type of abdominal wall hernias. It was claimed half a century ago that a general surgeon can only have one lumbar hernia repair experience in his or her career. ${ }^{[1]}$ Congenital hernias consist only less than $20 \%$ of all lumbar hernias. ${ }^{[1,2]}$ Among acquired lumbar hernias, secondary cases are less common than spontaneous ones. Although infections can cause lumbar hernias, most of the secondary hernias develop following surgical procedures or different types of traumas. ${ }^{[2]}$

Lumbar hernia repairs are usually difficult in comparison with typical midline hernias, because of anatomical characteristics of the region and lack of surgical experience. Many methods and technical modifications have been described to date however all the choices can be divided into two categories as open and laparoscopic. Suture repairs frequently result in recurrence due to tension, there- fore prosthetic materials have become the basis of the treatment. Lately, hybrid techniques have come into use by performing both open and laparoscopic approaches together. We herein present 3 consecutive cases of secondary lumbar hernias treated with hybrid repair by using intraperitoneal composite mesh.

\section{Case Report}

\section{Patients}

Three patients with secondary lumbar hernias admitted with the complaint of reducible lumbar mass following different etiologies within a one two-month period. Surgical treatments of three patient were performed with one week intervals.

Case 1 - A 65-year-old woman underwent an open right inguinal hernia repair with mesh 4 years ago. She devel- 


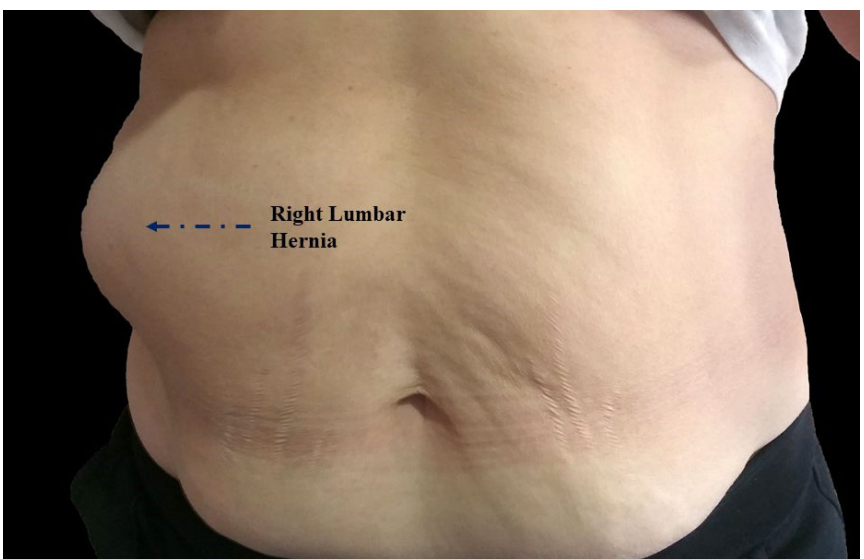

Figure 1. Preoperative upright photograph of Case\#2. Lumbar herniation is obvious.

oped a recurrence at the superior and the lateral of the repair line after a short while. She underwent two more mesh repairs. Both operations resulted in surgical site infection. Meshes were extracted possibly along with tissue losses. She developed a reducible mass in her right lumbar region. CT (computed tomography) revealed a lumbar hernia whereas no inguinal recurrence was detected (Fig. 1a). The patient admitted for definitive treatment of her hernia. She had only medically controlled arterial hypertension. Her BMI was 28.

Case 2 - A 48-year-old woman underwent abdominal liposuction 8 months ago. She complained of right lumbar pain and discomfort, however no specific diagnostic study was employed. Later she developed a swelling in her right lumbar region. A lumbar hernia was diagnosed in physical examination (Fig. 1). The patient admitted for diagnosis and treatment. CT revealed a lumbar herniation along with muscular atrophy in lateral abdominal muscle group (Fig. 2b). She had no systemic disorder. Her BMI was 25.

Case 3 - A 34-year-old military personnel had a traffic accident 2 years ago. Although a defect in the right lat-
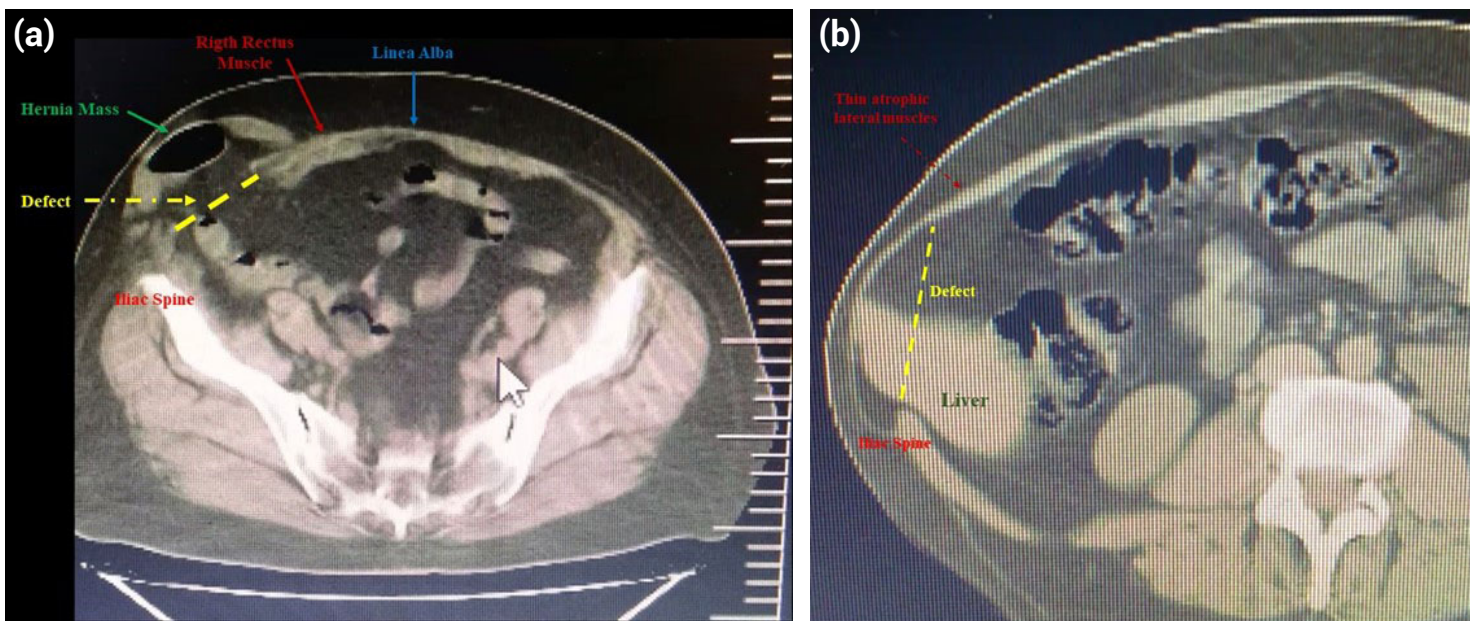

Figure 2. CT study of Case\#1 (a) and Case\#2

(b). (a) Low lumbar hernia containing intestinal loops. Case\#1. (b) Relatively higher lumbar hernia with no real hernia sac. Liver protruded through the hernia defect. Case\#2.
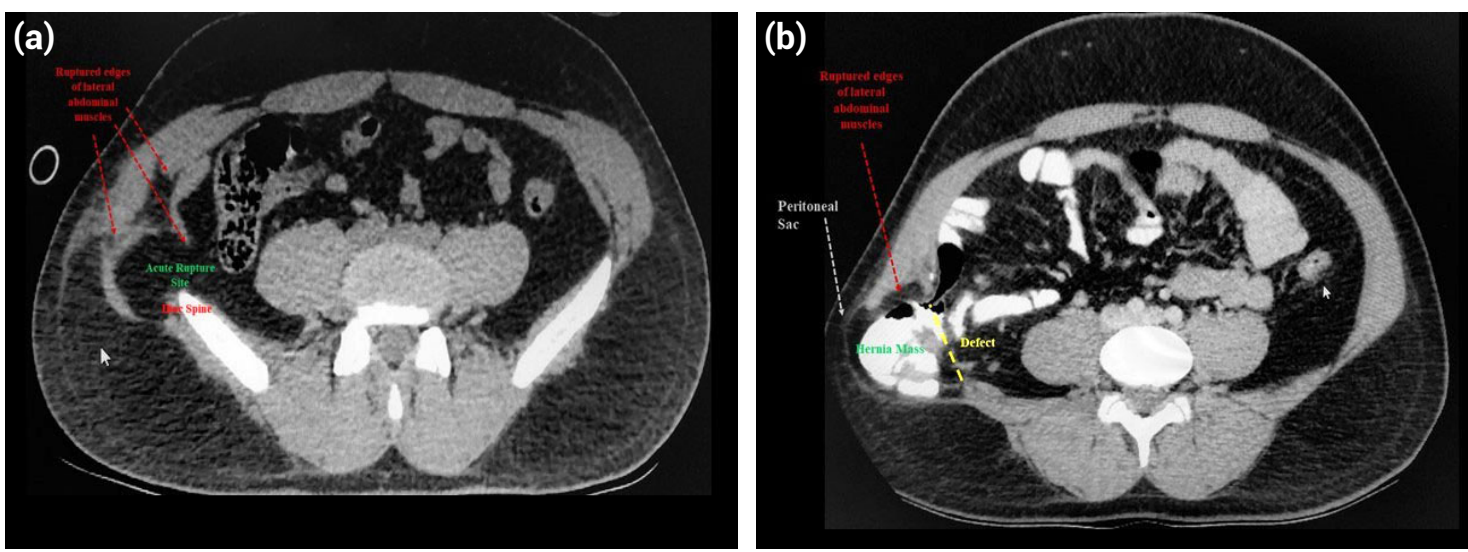

Figure 3. CT studies of Case\#3. (a) On the day of trauma. There is an obvious rupture in the lateral muscle group starting from just over the iliac spine. No organ herniation is seen at that time. (b) Following two consecutive failed suture repairs. Intestinal loops are herniated now. 


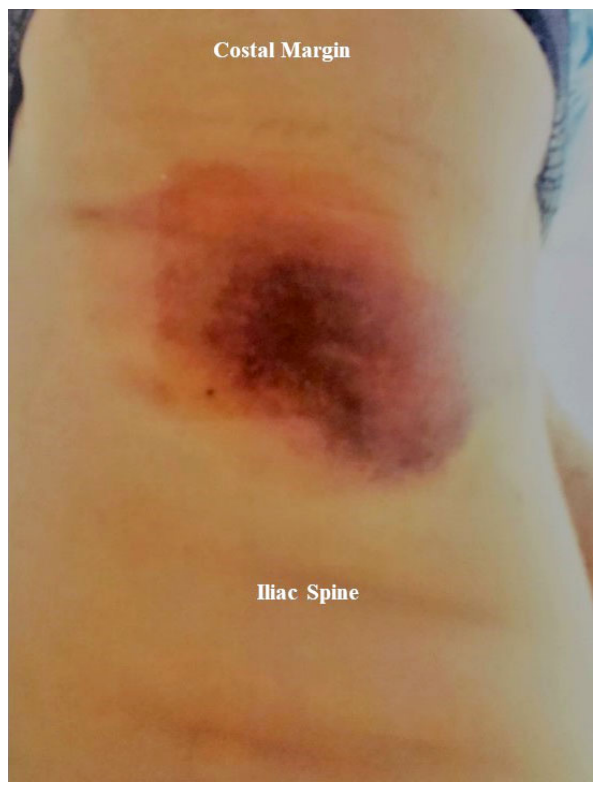

Figure 4. A wide ecchymotic area in the right lumbar region. The ecchymosis spreads between anterior and posterior axillary lines. The photograph was taken several days later the traffic accident. sac. Liver protruded through the hernia defect. Case\#2.

eral abdominal wall was seen in CT studied on that day, he had been observed without surgical intervention (Fig. 3a). A wide ecchymosis appeared in the right lumbar region several days after the accident (Fig. 4). He developed a symptomatic lumbar hernia soon and underwent two consecutive suture repairs 13 and 11 months ago. Early recurrences developed, and the patient admitted for a prosthetic repair. A new CT displayed a large defect and the herniation of intestinal loops (Fig. 3b). He had no systemic disease. His BMI was 29.

\section{Technique}

Defects in all three cases were relatively large and muscles were retracted, therefore laparoscopic defect closure might not be optimal. Thence combined laparoscopic and open repairs was planned for the patients. Antibiotic prophylaxis with $1 \mathrm{~g}$ intravenous cefazolin was administered. With the patient under general anesthesia, a urinary catheter was placed to decompress the bladder. Patients were placed in lateral decubitus position. Pneumoperitoneum was instituted with Veress needle on the left hypochondrium. A $15 \mathrm{~mm}$ incision was made for scope, and a $12 \mathrm{~mm}$ trocar and $30^{\circ}$ scope was introduced via the side of the abdomen. Two $5 \mathrm{~mm}$ trocars were inserted afterwards. The abdomen was explored, and the defect was seen on the right side (Fig. 5). Hernia content was reduced. Right paracolic peritoneum was incised. The borders of the hernia defect were fully exposed, and its sizes were measured with a soft ruler (Fig. 6). Afterwards the open part of the operation was commenced. Intraabdominal pressure due to pneumoperitoneum already displayed the exact location of the defect. Light source of the scope also helped (Fig. 7). A skin incision that is as short as possible was made over the defect $-6 \mathrm{~cm}$ in Cases \#1 and \#3, $8 \mathrm{~cm}$ in Case\#2-. Limited skin flaps were prepared in Case\#1 and Case\#3. Superior flap was advanced superiorly in Case\#3 to expose and plicate the luxated atrophic muscles. Retracted and folded muscles over the iliac crest are dissected carefully to obtain a larger musculo-aponeurotic flap for suture closure. Every effort was made to dissect and suture three layers of lateral abdominal muscles. 2/0 polydioxanone was used for muscle plication and defect closure (Fig. 8). Composite polyester mesh with an absorbable collagen barrier on the visceral side was used in all cases. At least a $5 \mathrm{~cm}$ overlap was provided. 0 No polydioxanone sutures were placed at the four edges of the mesh, and the mesh was inserted into the abdominal cavity through the open incision and sutures pulled out with a laparoscopic suture passer before closing the defect (Fig. 9). The inferior suture was passaged via a lowermost
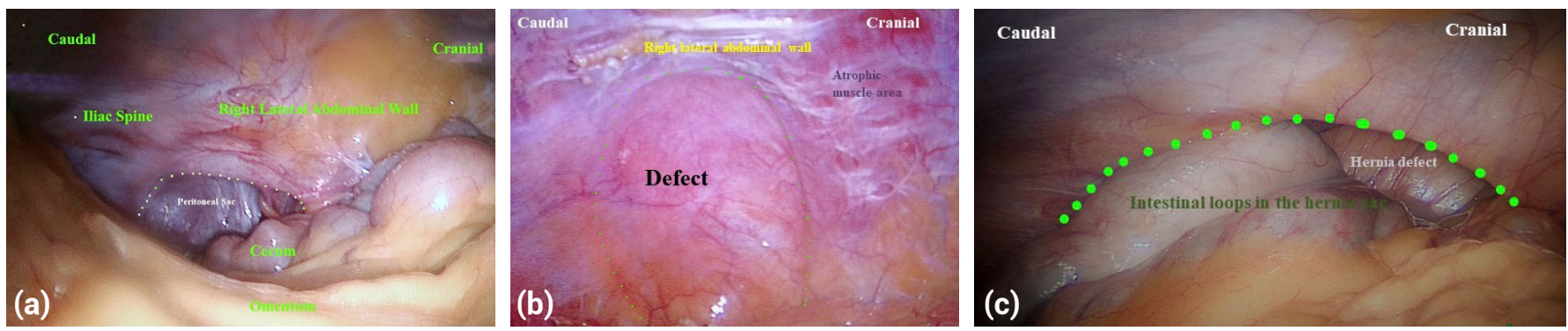

Figure 5. Operative views of the hernias from inside by the scope. (a) Small intestine loops are spontaneously reduced due to pneumoperitoneum. Cecum and the appendix vermiformis is seen in the hernia defect with loose adhesions. Case\#1. (b) Defect seems shallow. There is no real peritoneal sac. Liver is spontaneously reduced due to pneumoperitoneum. Torn endoabdominal fascia and atrophic muscles on the cranial part. Case\#2. (c) Intestinal loops are still in the hernia defect in spite of pneumoperitoneum. Case\#3. 


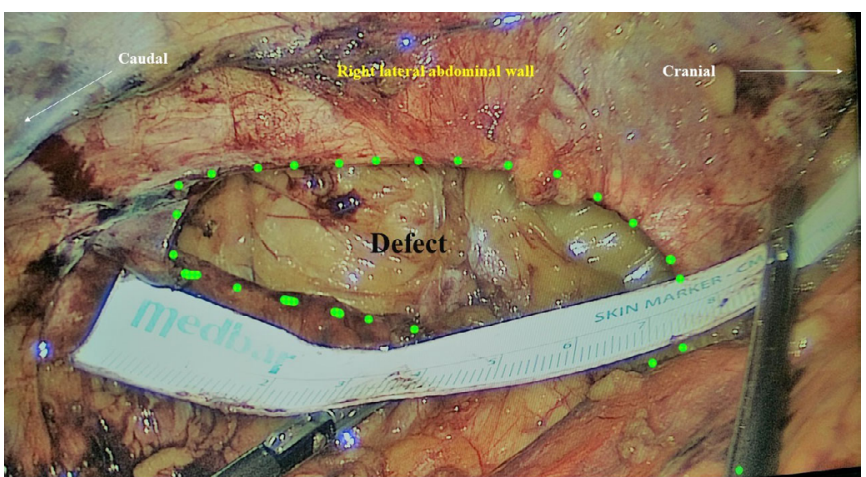

Figure 6. The borders of the hernia defect are exposed. A ruler is inserted into the abdominal cavity to measure the sizes of the defect. Case\#3.

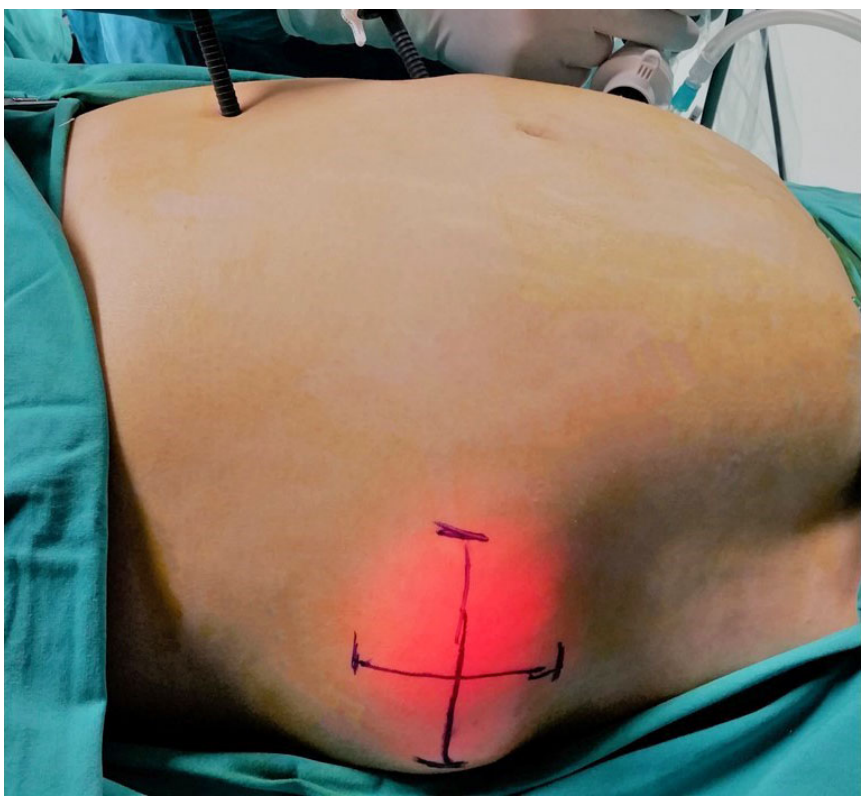

Figure 7. Although skin marking for open incision has been made before the surgery, the borders of the hernia is seen easily from outside with the aid of pneumoperitoneum. The light source of the scope also helps nicely.

point as close as possible to the iliac spine. Alternatively, mesh was inserted into the peritoneal cavity without any suture placement. Then pneumoperitoneum restored. Defect closure line was observed (Fig. 10). Additional external interrupted sutures were placed if gas leak was detected to obtain a tight closure as much as possible. Mesh was secured with tackers with double crown fixation technique; superiorly over the $10^{\text {th }}$ rib, inferiorly over the iliac crest periosteum, posteriorly on the musculus quadrates lumborum, and anteriorly abdominal muscles (Fig. 11). It is important to avoid entrapping the iliohypogastric and ilioinguinal nerves (Fig. 12). Finally, the abdomen was dessuflated, the incisions laparoscopic approaches were sutured. A vacuum drain was inserted beneath the skin flaps on the hernia site and the open incision was closed.
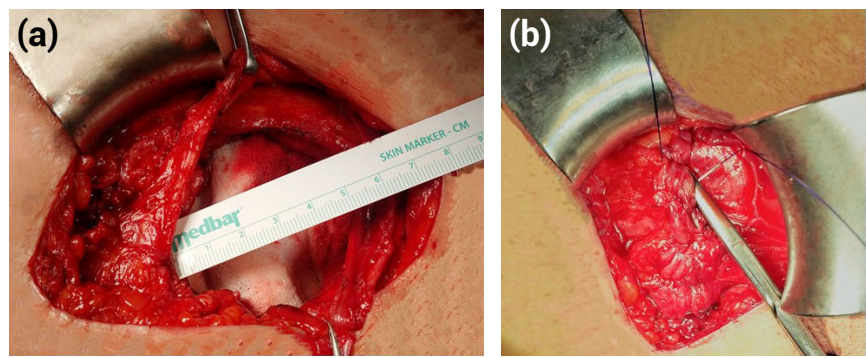

Figure 8. $(\mathbf{a}, \mathbf{b})$ Three layers of the lateral abdominal muscles are dissected free before closing the defect in Case\#2. Fascio-aponeurotic layers are sutured on separate rows. Composite mesh will be inserted into the abdominal cavity through $12 \mathrm{~mm}$ port.
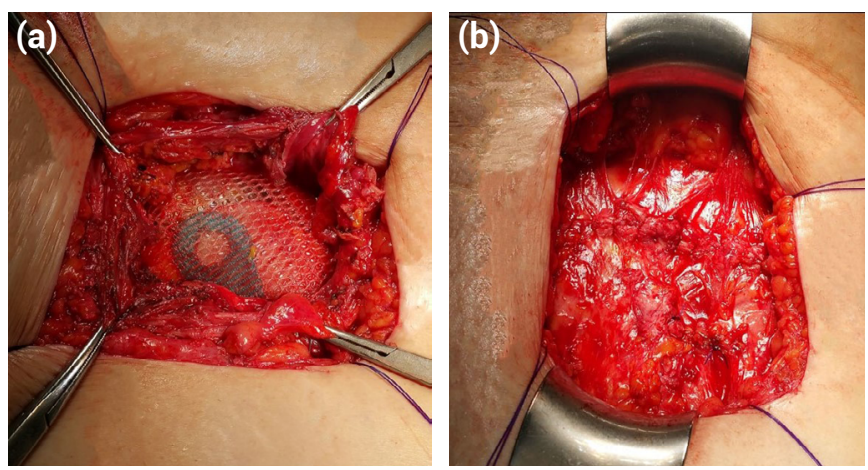

Figure 9. Mesh placement and defect closure in Case\# 1 . (a) Composite mesh is inserted into the abdominal cavity through the right lumbar incision of the open part of the operation. Pre-placed four threads are pulled with a laparoscopic suture passer without knotting. (b) Defect is closed with two-row continuous late absorbable suture.

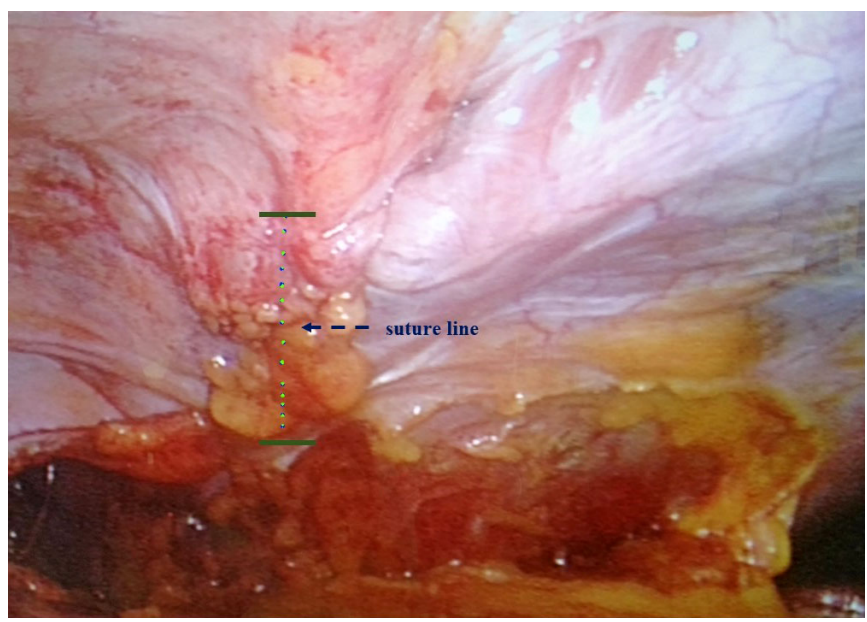

Figure 10. Suture line of the externally closed defect is seen from inside. No gap is detected.

The average operative time was 75 minutes. No intraoperative complications were recorded. No opioid analgesia was needed. All three patients were discharged on the $1^{\text {st }}$ postoperative day with oral analgesic prescription. A ten- 

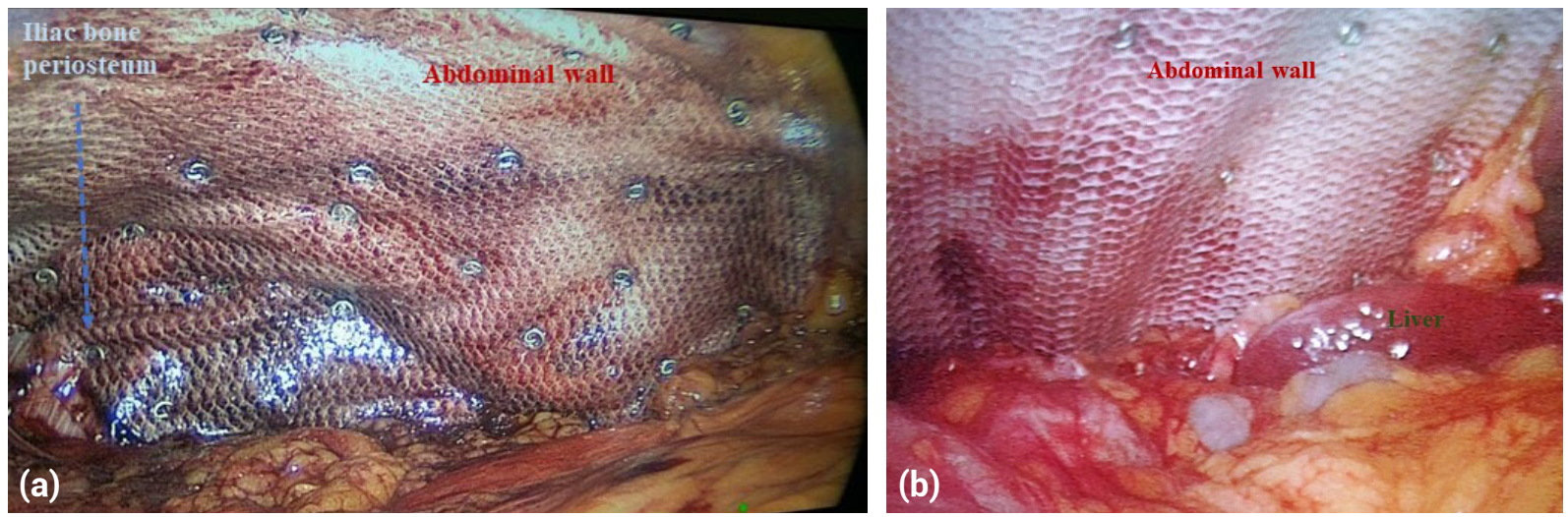

Figure 11. After tying the transfacial sutures, composite mesh is fixated with titanium tacks. (a) Inferior part of the mesh fixed on the iliac bone periosteum in Case\#3 in which two recurrences have developed earlier. (b) Small and large bowel loops are placed over the mesh after fixation on the musculus quadrates lumborum posteriorly. Liver which was herniated before now is just in neighborhood of the mesh.

day-long analgesic use was needed in Case\#3 which had a re-recurrent hernia. The drains were removed on the fifth postoperative day. No wound events or systemic complications were recorded in 30-day postoperative period (Fig. 13).

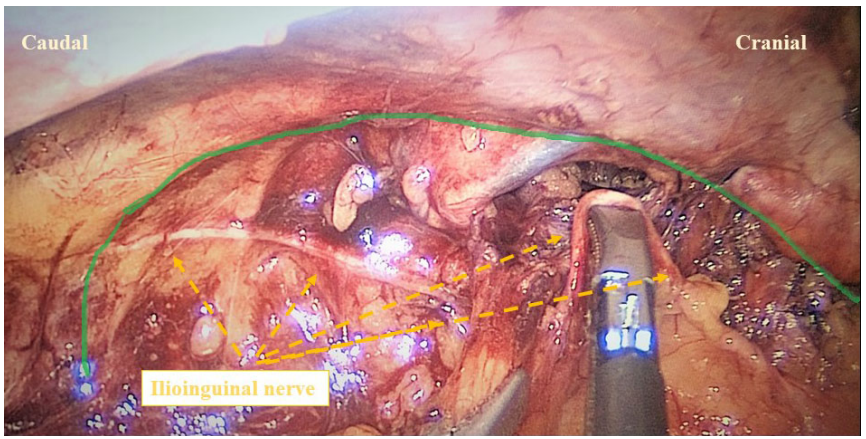

Figure 12. Ilioinguinal nerve is identified in hernia defect. This may especially be a problem when previous infectious processes and surgical repairs cause adhesion. The nerve dissected free carefully and taken out of the area of mesh fixation. Patient does not experience postoperative neurogenic pain. Case\#1.

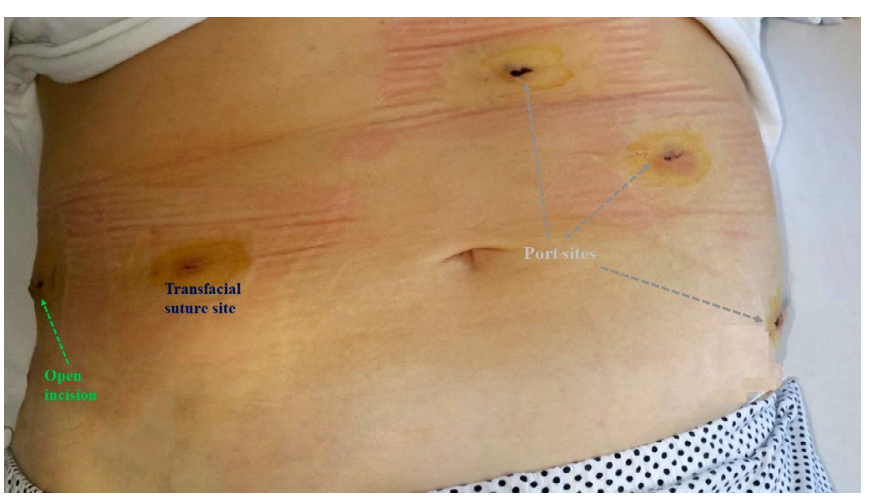

Figure 13. Postpoperative day-5 photograph of the Case\#2 on the supine position. Medial transfascial suture has been knotted at the lateral border of right rectus abdominis muscle.
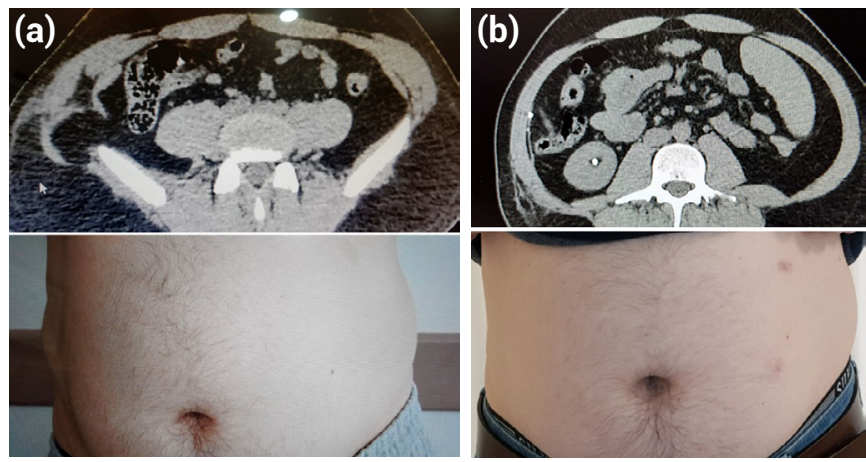

Figure 14. Comparisons of the gross appearance and computed tomography imaging of Case\#3 at preoperative period end postoperative year-1. (a) Preoperative. (b) Postoperative year-1.

After one year, none pf three cases developed any complication due to intraperitoneal onlay mesh placement or recurrence. Comparisons of the gross appearance and computed tomography imaging at preoperative period end postoperative year-1 are very satisfying (Fig. 14).

\section{Discussion}

Definition and terminology for lateral abdominal wall hernias differ in the publications in the literature. European Hernia Society (EHS) defines lateral hernias as those beyond the lateral margin of the rectal sheath. ${ }^{[3]}$ In the EHS Classification for lumbar hernia is the one that is located latero-dorsal of the anterior axillary line. The other hernia types in this subgroup are subcostal, flank and iliac. However, the terms "lumbar", "lateral" and "flank" are widely interchangeable with each other in the reports. ${ }^{[4]}$

The etiology of secondary lumbar hernias is mainly accumulated in two groups: surgical procedures and trauma. 
Nephrectomy is the typical surgery that may cause lateral incisional hernias. Hernias following nephrectomy are generally called as flank hernias. ${ }^{[3,4]}$ The mechanism of the herniation can be suture line failure or an injury to the subcostal nerve. Denervation also causes gradual weakening and thinning of the muscles which can eventually result in an apparent hernia. ${ }^{[2]}$ Other operations in the etiology are latissimus dorsi flaps for breast reconstruction or other purposes, resection of abdominal wall tumors, and iliac bone harvesting. ${ }^{[2,5]}$ Liposuction is a rare cause. To the best of our knowledge, there is no isolated lumbar hernia case report in the literature. However, abdominal organs perforations and peritonitis have been reported. ${ }^{[6,7]}$ The most unusual case report was described multiple bowel perforation and necrotising fasciitis after abdominal liposuction in a patient who already had bilateral lumbar hernias. ${ }^{[8]}$ In the present Case\#2, the patient developed a unilateral lumbar hernia several months after liposuction. This case seems to be unique to date. Moreno-Egea et al. ${ }^{[2]}$ defined lumbar hernias as a protrusion of preperitoneal or intra-abdominal contents into the lumbar area. There may not be a hernia sac. Indeed, this was the situation in Case\#2. Abdominal organs, including the edge of the right lobe the liver, herniated through a weak area of muscles. Peritoneal herniation was observed from inside with the scope however the peritoneum was also covered by a thin muscle layer.

Every type of trauma to the region may cause a lumbar hernia. Traffic accidents are common, ${ }^{[2,9]}$ but falls can be the reason. ${ }^{[9-11]}$ Animal hit has also been reported as the cause of lumbar hernias. ${ }^{[12]}$ Mechanism may be either direct blunt or penetrating trauma to the lumbar region or blast effect of blunt trauma to the other sites of the abdomen. Some traumatic lumbar hernias are diagnosed and repaired during acute trauma care ${ }^{[9,10]}$ or within several days following trauma when the patient is stable. ${ }^{[9,13]}$ An initial watchful waiting and subsequent elective repair is also possible. ${ }^{[14]}$

Generally, meshes are used in early open and laparoscopic repairs, ${ }^{[9,10,13]}$ however suture repair without any prosthetic patch has also been tried if there is no tissue loss. ${ }^{[15]} \mathrm{In}$ the present Case\#3, trauma had hit directly to the lumbar region. An acute herniation had been seen in immediate CT study however just left undiagnosed and untreated. Moreover, two consecutive suture repairs were performed with several-month intervals. Today mesh repairs are recommended according to experiences and principles of contemporary surgery. Laparoscopic mesh placement has better outcomes than open mesh repairs. ${ }^{[16]}$

Infection is a rare reason of lumbar hernias. Surgical site infections are sometimes result in incisional lumbar hernias, however lumbar hernia due to intraabdominal infection is very rare. ${ }^{[17]}$ Mesh infection and subsequent tissue loss due to mesh extraction and aggressive debridement was the causative factor in the present Case\#1. These events possibly converted a previous inguinal hernia to a more lateral hernia. Another possibility is that the patient might have a concomitant Spigelian hernia during previous repairs which considered a recurrent inguinal hernia and was treated with a typical onlay inguinal mesh repair. ${ }^{[18,19]}$ In the last operation we preferred laparoscopic mesh placement not only for this technique promises better result but also because of previous infection due to onlay mesh.

Laparoscopic repairs of lumbar hernias have been in use since late 1990's. ${ }^{[20,21]}$ Intraperitoneal and extraperitoneal mesh placement can be performed. ${ }^{[9,22,23]}$ There is no randomized clinical trial in the literature and the only clinical prospective study with long term follow-up states that laparoscopic repair lumbar hernia more efficient than open repair and can be considered the procedure of choice, whereas open repair may be better for treatment of the hernias larger than $15 \mathrm{~cm} .{ }^{[16]}$ However newer open techniques described by different authors promise good results. Veyrie et al. ${ }^{[24]}$ performed retromuscular polyester mesh placement following a wide dissection. The points for mesh fixation differed up to the exact location of the hernia, even the Cooper's ligament and the xyphoid process were used. They reported $4.9 \%$ recurrence rate after a median follow-up was 47 months. In fact, their technique was similar with the one described by the Aachen group where the mesh was placed between the external oblique muscle and the internal oblique muscle, which declared as the ideal place to position the mesh with adequate overlap. ${ }^{[25]}$ Very recently, Renard et al. ${ }^{[26]}$ reported a cohort of 31 patients who treated with retroperitoneal mesh placement. They used a Reverdin needle to fix the mesh far from the defect margins. In this technique, superior and inferior muscle flaps were superposed and sutured using U-stitches with slowly absorbable monofilament suture. The recurrence rate was $6.5 \%$ after a median follow-up of 27.5 months, with a $9.7 \%$ incidence of chronic pain. We also used to perform a similar open preperitoneal mesh repair for lateral hernias, however these re- 
pairs required longer operation time, very wide dissection area, occasional seroma formation, and longer time to return daily activity. For this reason, we preferred a hybrid technique that has all the advantages of open and laparoscopic approaches.

The first hybrid repair for abdominal wall hernias was described by Griniatsos et al. ${ }^{[27]}$ in 2009. The technique was used in the treatment of recurrent midline incisional hernias. Open part of the operation consisted of the reduction of the hernia sack content into the abdominal cavity after freeing all adhesions. A double-layer polytetrafluoroethylene mesh was inserted through the incision and fixed laparoscopically. No recurrences were detected after a maximum follow-up period of 12 months. Hybrid repairs can be a planned operative strategy or done when laparoscopic adhesiolysis was deemed unsafe and not feasible due to dense extensive adhesions of bowel to the abdominal wall. ${ }^{[28]}$ In other word, there are two scenarios. First, laparoscopic converted to open and then laparoscopic mesh fixation; second, open approach with laparoscopicassisted mesh fixation. ${ }^{[2]]}$

First report about the hybrid of lumbar hernias was published by Bathla in 2011. ${ }^{[14]}$ Two traumatic lumbar hernia cases were treated with interval repair by starting with laparoscopy for adhesiolysis and reduction of the hernia content. The redundant peritoneal sac was resected through an open incision and the mesh fixated by laparoscopic approach again. Inferior muscle flap was insufficient in one case where the mesh fixation required bone anchors to the iliac crest. In addition, atrophic muscle groups were restored with plication in one case to provide stronger repair and better cosmetic result. This is possibly one of the advantages of a hybrid technique against a solely laparoscopic approach. A similar technique was used in the present case series. Another potential benefit of the hybrid repair is providing a larger inferior muscle flap in secondary lumbar hernia cases. Trauma, surgery or infection usually cause shrinkage, retraction and folding in lateral muscles over the iliac crest. A meticulous dissection via an open approach can make an adequate inferior muscle flap for a better suture closure before laparoscopic mesh placement. Indeed, this let us to complete the repair with no need for bone anchoring by orthopedic surgeons, whom we already discussed the cases preoperatively with. In addition, peritoneal sac can advance into the interparietal tissue planes in these type of hernias, and an easier and more complete peritoneal sac resection is possible with open approach. This may lower the risk of postoperative seroma formation due to redundant hernia sac left in place. Another advantage of hybrid technique appears in patients with previous open mesh repairs. Only laparoscopic approach cannot give a chance to excise a migrated mesh or meshoma, but this becomes possible with open part of the combined repair. Moreover, it has been reported that adhesiolysis through a minimal open incision may be associated with a lower risk of undetected and untreated iatrogenic bowel injuries. ${ }^{[30]}$ Finally, open defect closure may be easier and more secure than laparoscopic suture placement in cases with large defects. Although there are some reports on laparoscopic mesh placement without defect closure, a recent meta-analysis has shown that defect closure significantly reduces seroma formation and other wound events and shortens duration of hospital stay. ${ }^{[31]}$

In conclusion, the present case series along with literature knowledge indicates that hybrid (combined laparoscopic and open) repair is feasible, safe and has certain potential advantages in treatment of secondary lumbar hernias.

\section{Disclosures}

Informed Consent: Written informed consent was obtained from the patients for the publication of the case report and the accompanying images.

Peer-review: Externally peer-reviewed.

Conflict of Interest: None declared.

\section{References}

1. Hafner CD, Wylie JH Jr, Brush BE. Petit's lumbar hernia: repair with Marlex mesh. Arch Surg 1963;86:180-6. [CrossRef]

2. Moreno-Egea A, Baena EG, Calle MC, Martínez JA, Albasini $\mathrm{JL}$. Controversies in the current management of lumbar hernias. Arch Surg 2007;142:82-8. [CrossRef]

3. Muysoms FE, Miserez M, Berrevoet F, Campanelli G, Champault GG, Chelala $E$, et al. Classification of primary and incisional abdominal wall hernias. Hernia 2009;13:407-14.

4. Zhou DJ, Carlson MA. Incidence, etiology, management, and outcomes of flank hernia: review of published data. Hernia 2018;22:353-361. [CrossRef]

5. Varban 0 . Lumbar hernia after breast reconstruction. Int J Surg Case Rep 2013;4:869-71. [CrossRef]

6. Di Candia M, Malata CM. Aesthetic and functional abdominal wall reconstruction after multiple bowel perforations secondary to liposuction. Aesthetic Plast Surg 2011;35:274-7.

7. Zakine G, Baruch J, Dardour JC, Flageul G. Perforation of viscera, a dramatic complication of liposuction: a review of 19 cases evaluated by experts in France between 2000 and 
2012. Plast Reconstr Surg 2015;135:743-50. [CrossRef]

8. Dellière $V$, Bertheuil $N$, Harnois $Y$, Thiénot $S$, Gérard $M$, Robert $M$, et al. Multiple bowel perforation and necrotising fasciitis secondary to abdominal liposuction in a patient with bilateral lumbar hernia. Indian J Plast Surg 2014;47:436-40.

9. Chan K, Towsey K, Cavallucci D, Green B. Traumatic lumbar hernia repair: experience at the Royal Brisbane and Women's Hospital. Hernia 2017;21:317-322. [CrossRef]

10. den Hartog D, Tuinebreijer WE, Oprel PP, Patka P. Acute traumatic abdominal wall hernia. Hernia 2011;15:443-5. [CrossRef]

11. Uzun MA, Köksal N, Onur E, Günerhan Y, Sahin UY, Celik A. Traumatic lumbar hernia. Ulus Travma Acil Cerrahi Derg 2008;14:253-5.

12. Singh $B$, Kumar $A$, Kaur $A$, Singla RL. Bullhorn hernia: a rare traumatic abdominal wall hernia. Niger J Surg 2015;21:63-5.

13. Kaminski S, Diamond S. The early laparoscopic repair of a traumatic lumbar hernia: safe and successful. J Surg Case Rep 2017;2017:rjx188.

14. Bathla L, Davies E, Fitzgibbons RJ Jr, Cemaj S. Timing of traumatic lumbar hernia repair: is delayed repair safe? Report of two cases and review of the literature. Hernia 2011;15:205-9.

15. Saboo SS, Khurana B, Desai N, Juan YH, Landman W, Sodickson A, et al. Traumatic lumbar hernia: can't afford to miss. Emerg Radiol 2014;21:325-7. [CrossRef]

16. Moreno-Egea A, Alcaraz AC, Cuervo MC. Surgical options in lumbar hernia: laparoscopic versus open repair. A long-term prospective study. Surg Innov 2013;20:331-44. [CrossRef]

17. Ali SM, Subramaniam S. Appendicular abscess as an unprecedented cause of an inferior lumbar hernia. Ann R Coll Surg Engl 2017;99:e85-e87. [CrossRef]

18. Matsui S, Nitori N, Kato A, Ikeda Y, Kiatagwa $Y$, Hasegawa $H$, et al. Laparoscopic totally extra-peritoneal hernia repair for bilateral Spigelian hernias and coincident inguinal hernia: A case report. Int J Surg Case Rep 2016;28:169-172. [CrossRef]

19. Kılıç MÖ, Değirmencioğlu G, Dener C. A rare case of Spigelian hernia combined with direct and indirect inguinal hernias. Turk J Surg 2015;33:40-42. [CrossRef]

20. Burick AJ, Parascandola SA. Laparoscopic repair of a traumatic lumbar hernia: a case report. J Laparoendosc Surg
1996;6:259-62. [CrossRef]

21. Heniford BT, lannitti DA, Gagner M. Laparoscopic inferior and superior lumbar hernia repair. Arch Surg 1997;132:1141-4.

22. Sun J, Chen X, Li J, Zhang Y, Dong F, Zheng M. Implementation of the trans-abdominal partial extra-peritoneal (TAPE) technique in laparoscopic lumbar hernia repair. BMC Surg 2015;15:118. [CrossRef]

23. Yavuz N, Ersoy YE, Demirkesen O, Tortum OB, Erguney S. Laparoscopic incisional lumbar hernia repair. Hernia 2009;13:281-6. [CrossRef]

24. Veyrie N, Poghosyan T, Corigliano N, Canard G, Servajean S, Bouillot JL. Lateral incisional hernia repair by the retromuscular approach with polyester standard mesh: topographic considerations and long-term follow-up of 61 consecutive patients. World J Surg 2013;37:538-44. [CrossRef]

25. Stumpf $M$, Conze J, Prescher A, Junge K, Krones CJ, Klinge $U$, et al. The lateral incisional hernia: anatomical considerations for a standardized retromuscular sublay repair. Hernia 2009;13:293-7. [CrossRef]

26. Renard Y, de Mestier L, Cagniet A, Demichel N, Marchand C, Meffert JL, et al. Open retromuscular large mesh reconstruction of lumbar incisional hernias including the atrophic muscular area. Hernia 2017;21:341-349. [CrossRef]

27. Griniatsos J, Yiannakopoulou E, Tsechpenakis A, Tsigris C, Diamantis T. A hybrid technique for recurrent incisional hernia repair. Surg Laparosc Endosc Percutan Tech 2009;19:e17780. [CrossRef]

28. Ji Y, Zhan X, Wang Y, Zhu J. Combined laparoscopic and open technique for the repair of large complicated incisional hernias. Surg Endosc 2013;27:1778-83. [CrossRef]

29. Stoikes N, Quasebarth M, Brunt LM. Hybrid ventral hernia repair: technique and results. Hernia 2013;17:627-32. [CrossRef]

30. Ahonen-Siirtola M, Rautio T, Biancari F, Ohtonen P, Mäkelä J. Laparoscopic versus Hybrid Approach for Treatment of Incisional Ventral Hernia. Dig Surg 2017;34:502-506. [CrossRef]

31. Tandon A, Pathak S, Lyons NJ, Nunes QM, Daniels IR, Smart NJ. Meta-analysis of closure of the fascial defect during laparoscopic incisional and ventral hernia repair. Br J Surg 2016;103:1598-1607. [CrossRef] 